The Astrophysical Journal, 651:667-675, 2006 November 10

(C) 2006. The American Astronomical Society. All rights reserved. Printed in U.S.A.

\title{
THREE GRAVITATIONAL LENSES FOR THE PRICE OF ONE: ENHANCED STRONG LENSING THROUGH GALAXY CLUSTERING ${ }^{1}$
}

\author{
C. D. Fassnacht, ${ }^{2}$ J. P. McKean, ${ }^{2}$ L. V. E. Koopmans, ${ }^{3}$ T. Treu, ${ }^{4}$ R. D. Blandford, ${ }^{5}$ M. W. Auger, ${ }^{2}$ \\ T. E. Jeltema, ${ }^{6}$ L. M. Lubin, ${ }^{2}$ V. E. Margoniner, ${ }^{2}$ and D. Wittman ${ }^{2}$ \\ Received 2006 March 16; accepted 2006 July 9
}

\begin{abstract}
We report the serendipitous discovery of two strong gravitational lens candidates (ACS J160919+6532 and ACS J160910+6532) in deep images obtained with the Advanced Camera for Surveys on the Hubble Space Telescope, each less than $40^{\prime \prime}$ from the previously known gravitational lens system CLASS B1608+656. The redshifts of both lens galaxies have been measured with Keck and Gemini: one is a member of a small galaxy group at $z \approx 0.63$ that also includes the lensing galaxy in the B1608+656 system, and the second is a member of a foreground group at $z \approx 0.43$. By measuring the effective radii and surface brightnesses of the two lens galaxies, we infer their velocity dispersions based on the passively evolving fundamental plane (FP) relation. Elliptical isothermal lens mass models are able to explain their image configurations within the lens hypothesis, with a velocity dispersion compatible with that estimated from the FP for a reasonable source-redshift range. Based on the large number of massive early-type galaxies in the field and the number density of faint blue galaxies, the presence of two additional lens systems around CLASS B1608+656 is not unlikely in hindsight. Gravitational lens galaxies are predominantly early-type galaxies, which are clustered, and the lensed quasar host galaxies are also clustered. Therefore, obtaining deep high-resolution images of the fields around known strong-lens systems is an excellent method of enhancing the probability of finding additional strong gravitational lens systems.
\end{abstract}

Subject headings: galaxies: individual (B1608+656) — gravitational lensing

\section{INTRODUCTION}

Strong gravitational lenses are excellent tools for cosmological and astrophysical studies (see, e.g., Kochanek 2006). Because of their utility, an increase in the number of lenses is a highly desirable goal, especially when the numbers become large enough to marginalize over hidden parameters associated with any given lens system. The majority of lenses discovered in the last decade were found through dedicated surveys that used a variety of techniques to find strong lenses, such as targeting the potential lensed objects (e.g., Bahcall et al. 1992; Myers et al. 2003; Browne et al. 2003; Winn et al. 2000; Wisotzki et al. 2002; Pindor et al. 2004), searching around the potential lensing galaxies (e.g., Fassnacht et al. 2004), or looking for multiple redshifts associated with a single object in large spectroscopic surveys (e.g., Bolton et al. 2004, 2005, 2006; Willis et al. 2006). However, a large number of lens systems were discovered serendipitously. In this paper we report just such a discovery. We have found two additional strong lens candidates in a single image centered on a known gravitational lens, the data of which were obtained with the Advanced Camera for Surveys (ACS; Ford et al. 1998, 2003) on the Hubble Space Telescope (HST).

\footnotetext{
${ }^{1}$ Based in part on observations made with the NASA/ESA Hubble Space Telescope, obtained at the Space Telescope Science Institute, which is operated by the Association of Universities for Research in Astronomy, Inc., under NASA contract NAS 5-26555. These observations are associated with program GO-10158.

2 Department of Physics, University of California, 1 Shields Avenue, Davis, CA 95616; fassnacht@physics.ucdavis.edu.

3 Kapteyn Astronomical Institute, University of Groningen, P.O. Box 800, 9700 AV Groningen, Netherlands.

4 Department of Physics, University of California, Broida Hall, Building 572, Santa Barbara, CA 93106.

5 Kavli Institute for Particle Astrophysics and Cosmology, Stanford Linear Accelerator Center, MS 75, 2575 Sand Hill Road, Menlo Park, CA 94025.

${ }^{6}$ Observatories of the Carnegie Institute of Washington, 813 Santa Barbara Street, Pasadena, CA 91101.
}

The main goal of the ACS observations was to obtain a highsensitivity image of the Einstein ring of a previously known gravitational lens, CLASS B1608+656 (Myers et al. 1995). The Einstein ring provides further constraints to the lensing mass model and reduces the uncertainties in the determination of $H_{0}$ from this time delay system (e.g., Kochanek et al. 2001; Koopmans et al. 2003). The exquisite angular resolution and surface brightness sensitivity of the ACS imaging also allow the properties of the B1608+656 field to be studied. In particular, the contribution to the B1608+656 image splitting by a small group of galaxies associated with the main lensing galaxy has been investigated (Fassnacht et al. 2006), and a weak-lensing analysis of the mass distribution along the line of sight to B1608+656 is being conducted. The deep ACS imaging has also allowed us to find two additional galaxy-scale gravitational lens systems in the same field of view (FOV).

In $\S 2$ we present the ACS and ground-based imaging of the two lens candidates. The spectra of the lensing galaxies and one of the lensed sources are presented in $\S 3$. We test lensing mass models for the two systems using a nonparametric lensing code in $\S 4$. Finally, in $\S 5$ we discuss the lensing hypothesis for both systems and estimate the likelihood of finding two additional lenses in this field. Throughout this paper we assume a flat universe with $\Omega_{M}=0.3, \Omega_{\Lambda}=0.7$, and, unless otherwise stated, we express the Hubble constant as $H_{0}=100 \mathrm{hm} \mathrm{s}^{-1} \mathrm{Mpc}^{-1}$.

\section{OPTICAL IMAGING}

In this section we present space- and ground-based multicolor optical imaging of the two lens candidates in the field of B1608+656.

\subsection{Hubble Space Telescope}

High-resolution optical imaging of the B1608+656 field was obtained with the ACS (GO-10158; PI: Fassnacht). The data were acquired over the course of five visits between 2004 August 24 
TABLE 1

IMAGING OBSERVATIONS

\begin{tabular}{ccccr}
\hline \hline Date & Telescope & Instrument & Filter & $\begin{array}{c}t_{\text {exp }} \\
(\mathrm{s})\end{array}$ \\
\hline 2000 Apr..................... & P60 & CCD13 & $g$ & 7200 \\
& P60 & CCD13 & $r$ & 3600 \\
& P60 & CCD13 & $i$ & 3000 \\
2000 Jul..................... & P60 & CCD13 & $g$ & 5400 \\
2004 Aug/Sep ............... & $H S T$ & ACS/WFC & F606W & 22516 \\
& $H S T$ & ACS/WFC & F814W & 28144 \\
\hline
\end{tabular}

and September 17. The Wide Field Channel (WFC) of the ACS was used throughout, providing an FOV of $202^{\prime \prime} \times 202^{\prime \prime}$ and a scale of 0.05 pixel $^{-1}$. Our observations consisted of nine orbits that used the F606W filter and 11 orbits with the F814W filter, corresponding to total exposure times of 22,516 and 28,144 s, respectively. The data were reduced in the standard manner using the stsdas package within IRAF. ${ }^{7}$ The final combined images were produced using multidrizzle (Koekemoer et al. 2002), which also corrected the data for the ACS geometric distortion. The area covered by the final combined image in each filter, defined as the region in which the weight file had pixel values greater than 2000, is $11.9 \mathrm{arcmin}^{2}$. Catalogs of objects in the ACS images were generated by running SExtractor (Bertin \& Arnouts 1996) with the parameters suggested by Benitez et al. (2004). The count rates in the images were converted to Vegabased magnitudes using the zero points on the ACS Web site. ${ }^{8}$ Full details of the acquisition and reduction of our ACS imaging

7 IRAF (Image Reduction and Analysis Facility) is distributed by the National Optical Astronomy Observatory, which is operated by the Association of Universities for Research in Astronomy, Inc., under cooperative agreement with the National Science Foundation.

${ }^{8}$ See http://www.stsci.edu/hst/acs/analysis/zeropoints.

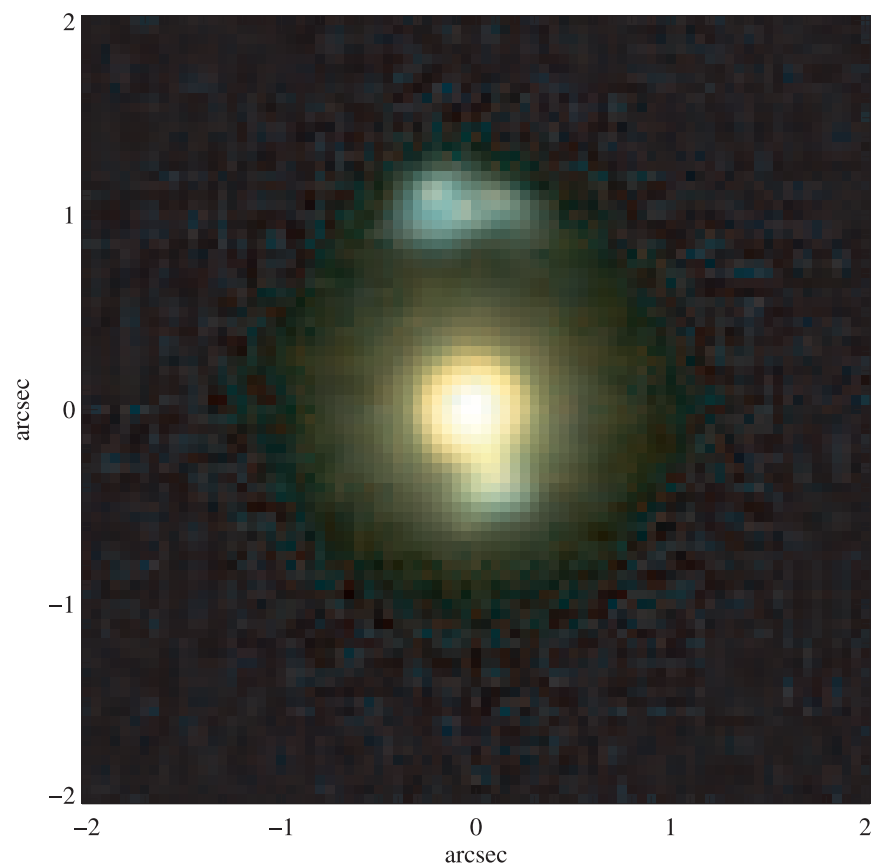

will be presented in a future paper. A summary of the imaging observations is given in Table 1.

A visual inspection of the ACS images, undertaken with the goal of evaluating the properties of the galaxies surrounding B1608+656, revealed two objects with lenslike morphologies. Each consists of a reddish early-type galaxy with a nearby blue arc or multiple blue blobs, similar to other lenses found in $H S T$ imaging (e.g., Ratnatunga et al. 1999; Fassnacht et al. 2004; Blakeslee et al. 2004; Bolton et al. 2006). The two lens candidates, ACS J160919+6532 and ACS J160910+6532, are shown in Figure 1. For simplicity, the two lens candidates are hereafter referred to as "Fred" and "Ginger," respectively. In Figure 2 we show a larger FOV that includes the two lensing candidates and $\mathrm{B} 1608+656$. Both lens candidates are in close proximity to B1608+656 on the sky: Fred lies $\sim 36^{\prime \prime}$ to the northeast, whereas Ginger is $\sim 37^{\prime \prime}$ to the north-northwest. The coordinates of the lens candidates are given in Table 2.

Fred consists of a red spheroidal galaxy with two blue candidate lensed images to the north and south. The blue image to the north is extended in an east/west direction, while the southern blue image both is fainter and covers a smaller angular size on the sky. The sizes and surface brightnesses of the two blue images are consistent with gravitational lensing. Their separation is $\sim 1 " .5$.

The second lens candidate, Ginger, is a bright red elliptical with an extended blue gravitational arc to the northeast. The narrow arclike feature appears to curve toward the red galaxy, which is consistent with lensing. There is also evidence of substructure in the arc, which is presumably due to clumps of star formation. No obvious counterimage can visually be identified in the color map. However, the galaxy-subtracted residuals (§ 4) do show a faint possible counterimage.

The photometric properties of the lens candidates are listed in Table 3. We used the GALFIT package (Peng et al. 2002) to model the lensing galaxies ("lenses") and obtained the lens galaxy magnitudes by running SExtractor on the GALFIT model

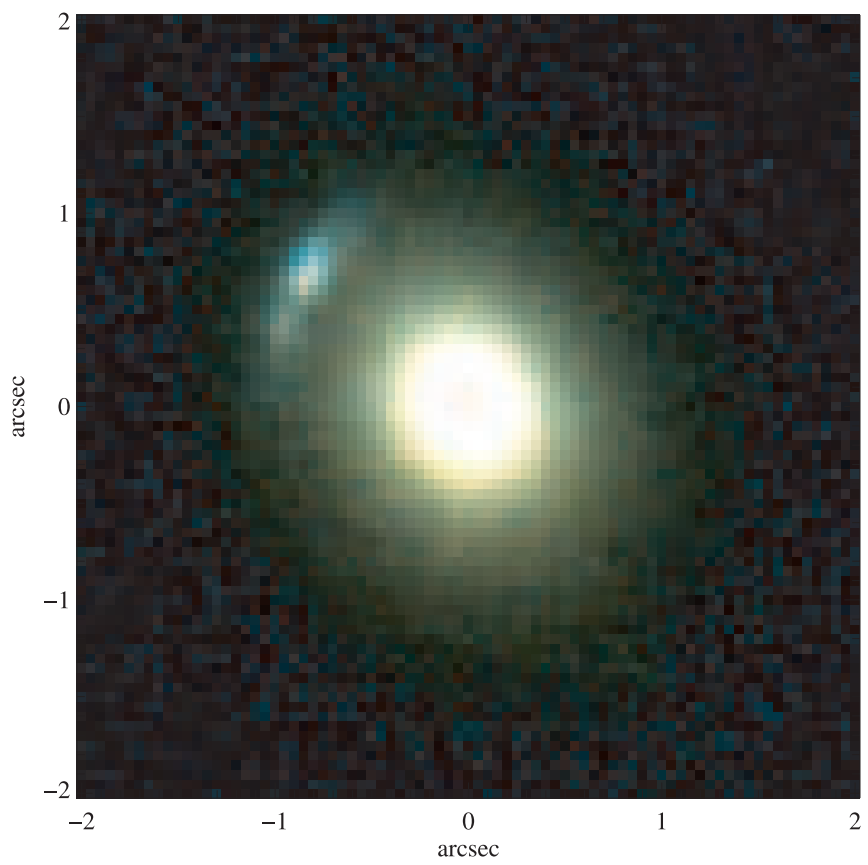

FIG. 1. - Three-color images of Fred (left) and Ginger (right). The images were constructed from the ACS images, with the F606W data in the blue channel, the sum of

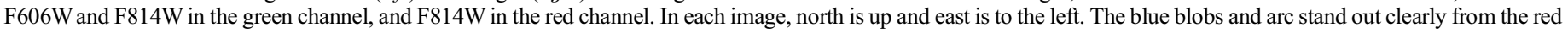
lensing galaxies. 


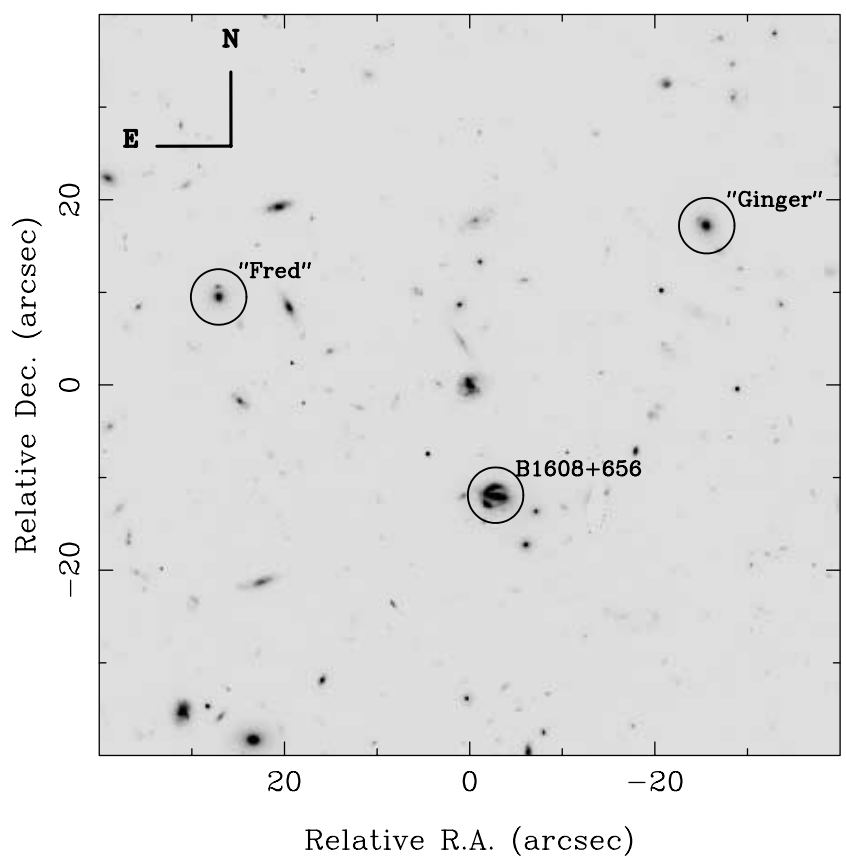

FIG. 2.-Wider FOV, showing B1608+656 and the two additional strong-lens candidates in relation to it. The image was obtained through the F814W filter with the ACS.

image. The photometry of the lensed images ("sources") was measured by running SExtractor on data from which the lens galaxy emission had been subtracted, that is, the residual images produced by GALFIT. In each case, the value for the lensed source in the table refers to the brighter of the two lensed images. Two apertures were used in the photometry. The first is that defined by the MAG_AUTO algorithm in SExtractor, with the default values for the Kron factor and minimum radius. The second is a circular aperture with a radius of $1^{\prime \prime}$. We estimate that the systematic uncertainties in the HST photometry are $\sim 0.05 \mathrm{mag}$. The errors on the lens magnitudes are dominated by systematic uncertainties, which are $\sim 20$ times larger than the formal statistical errors. In contrast, the statistical uncertainties dominate for the source magnitudes.

We determined structural parameters of the lensing galaxies by fitting de Vaucouleurs profiles to the galaxies after masking the lensed features, following the procedures described in Treu et al. (2006). We also used the GALFIT package (Peng et al. 2002). The effective radii $\left(R_{e}\right)$, axis ratios $\left(q_{*}=b / a\right)$, and major-axis position angles $(\theta)$ are given in Table 4 . Note that the values of $\theta$ given for Fred are essentially meaningless since the light distribution is nearly perfectly circular. To transform the observed quantities into rest-frame values, we follow the procedure described in Treu et al. (2001) while setting $h=0.7$. At the redshifts of the lensing galaxies, the effective radii are $R_{e}=4.8 \pm 0.5$ and $3.6 \pm 0.4 \mathrm{kpc}$ for Fred and Ginger, respectively. To compute the rest-frame $B$-band surface brightnesses, we first correct the $H S T$ photometry in the band closest to the rest-frame $B$ band for Galactic extinction. For Fred we use the observed F814W band, while for Ginger we use the F606W band. The transformation to rest-frame $B$ employed a $K$-correction and a compensation for surface brightness dimming. The $K$-correction and the related uncertainties are computed from the average and rms scatter obtained with a set of stellar synthesis models (Bruzual \& Charlot 1993) that reproduce the observed colors within the errors. The resulting effective surface brightnesses for Fred and Ginger are $\mathrm{SB}_{e}=20.42 \pm 0.05$ and $20.81 \pm 0.05 \mathrm{mag} \operatorname{arcsec}^{-2}$, respectively. Correcting for evolution to $z=0$, adopting $d \log \left(M / L_{B}\right) / d z=-0.72 \pm 0.04$ (Treu
TABLE 2

Lens System Coordinates

\begin{tabular}{ccccc}
\hline \hline Name & $\begin{array}{c}\text { R.A. } \\
(\mathrm{J} 2000.0)\end{array}$ & $\begin{array}{c}\text { Decl. } \\
(\mathrm{J} 2000.0)\end{array}$ & $z_{\text {lens }}$ \\
\hline Fred ................................................... & 160918.760 & +653249.72 & 0.6321 \\
Ginger..... & 160910.292 & +653257.38 & 0.4264 \\
\hline
\end{tabular}

Note.-Units of right ascension are hours, minutes, and seconds, and units of declination are degrees, arcminutes, and arcseconds.

et al. 2005), and assuming that the lens galaxies obey the fundamental plane (FP; Dressler et al. 1987; Djorgovski \& Davis 1987) relationship yield estimates for the central velocity dispersions of $\sigma_{\mathrm{FP}}=180 \pm 50$ and $140 \pm 30 \mathrm{~km} \mathrm{~s}^{-1}$ for Fred and Ginger, respectively. The uncertainty on $\sigma_{\mathrm{FP}}$ is dominated by the uncertainty in the evolutionary correction and in the intrinsic thickness of the FP (assumed to be 0.08 in $\log R_{e}$ ).

\subsection{Palomar 60 inch Telescope}

Ground-based imaging of the B1608+656 field was obtained as part of a program to investigate the environments of strong gravitational lenses (e.g., Fassnacht et al. 2006). The observations were conducted using the Palomar 60 inch $(1.5 \mathrm{~m})$ Mayer telescope (P60), with the CCD13 detector. The CCD provides an FOV of approximately $13^{\prime} \times 13^{\prime}$, with a pixel size of 0.379 . The data were taken using the Gunn $g, r$, and $i$ filters in 2000 April. The seeing was approximately 1".2 through the $r$ and $i$ filters, while it was 1".5 through the $g$ filter. Exposure times are given in Table 1. A further observing session in 2000 July was used to increase the depth of the $g$-band data. In these observations, the seeing was $\sim 1$ '. 2 . The conditions during both observing sessions were photometric. The data were reduced using standard IRAF tasks, and then the $g$-band data from the two observing sessions were combined. Astrometric solutions were computed with the aid of positions obtained from the USNO-A2.0 catalog (Monet et al. 1998), while photometric solutions were derived from observations of several Gunn standard stars (Thuan \& Gunn 1976). Given the seeing and the faintness of the background objects, the background sources are not resolved in the P60 imaging. Therefore, only total (lens+source) magnitudes are given in Table 3. The SExtractor MAG_AUTO magnitudes were computed with the default settings for the Kron factor and minimum radius, while the circular apertures had radii of $2^{\prime \prime}$. Once again, the systematic errors in the determination of the magnitudes are much larger than the formal statistical errors, and the uncertainties given in the table reflect our estimates of the calibration errors.

\section{SPECTROSCOPY AND REDSHIFTS}

Several attempts to obtain the redshifts of the lenses and background sources have been made. A summary of the spectroscopic observations is given in Table 5. The first observation, with the Echellete Spectrograph and Imager (ESI; Sheinis et al. 2002) on the W. M. Keck II Telescope, was obtained as a part of the general investigation of the environment of the B1608+656 lens system. The subsequent observations, with the Low Resolution Imaging Spectrograph (LRIS; Oke et al. 1995) at Keck and the Gemini Multi-Object Spectrograph (GMOS; Hook et al. 2004) on GeminiNorth, were targeted specifically at the lens candidates. The LRIS observations were obtained on subsequent nights and consisted of one exposure on each lens candidate. Each observation was taken at the very end of the night, ending after $18^{\circ}$ twilight, so the blue-side observations were swamped by the sky emission. In contrast, the GMOS observations of Fred were obtained in 
TABLE 3

Lens System Photometric Properties

\begin{tabular}{|c|c|c|c|c|c|c|c|c|}
\hline Name & Aperture & $g_{\text {tot }}$ & $r_{\text {tot }}$ & $i_{\text {tot }}$ & F606W $W_{\text {lens }}$ & $\mathrm{F} 814 \mathrm{~W}_{\text {lens }}$ & F606W $\mathrm{W}_{\text {src }}$ & $\mathrm{F} 814 \mathrm{~W}_{\mathrm{src}}$ \\
\hline \multirow[t]{2}{*}{ Fred ................... } & MAG_AUTO & $22.5 \pm 0.1$ & $21.6 \pm 0.1$ & $21.0 \pm 0.1$ & $22.00 \pm 0.05$ & $20.20 \pm 0.05$ & $23.9 \pm 0.3$ & $23.1 \pm 0.4$ \\
\hline & Circular & $22.7 \pm 0.1$ & $21.7 \pm 0.1$ & $21.1 \pm 0.1$ & $22.31 \pm 0.05$ & $20.46 \pm 0.05$ & $24.0 \pm 0.3$ & $23.1 \pm 0.4$ \\
\hline \multirow[t]{2}{*}{ Ginger.................. } & MAG_AUTO & $22.1 \pm 0.1$ & $20.8 \pm 0.1$ & $20.4 \pm 0.1$ & $21.21 \pm 0.05$ & $19.84 \pm 0.05$ & $24.6 \pm 0.5$ & $24.0 \pm 0.6$ \\
\hline & Circular & $22.4 \pm 0.1$ & $21.1 \pm 0.1$ & $20.7 \pm 0.1$ & $21.49 \pm 0.05$ & $20.09 \pm 0.05$ & $24.6 \pm 0.5$ & $24.1 \pm 0.6$ \\
\hline
\end{tabular}

NoTES._- The magnitudes in the table are not corrected for Galactic extinction. The MAG_AUTO magnitudes are calculated with the SExtractor default values for the Kron factor and minimum radius. The circular apertures have radii of $2^{\prime \prime}$ for the ground-based magnitudes and $1^{\prime \prime}$ for $H S T$ magnitudes.

TABLE 4

Lensing Galaxy Structural Properties

\begin{tabular}{|c|c|c|c|c|c|c|}
\hline Name & $\begin{array}{l}R_{e, \mathrm{~F} 606 \mathrm{~W}} \\
(\operatorname{arcsec})\end{array}$ & $\begin{array}{l}R_{e, \mathrm{~F} 814 \mathrm{~W}} \\
(\operatorname{arcsec})\end{array}$ & $q_{*, \mathrm{~F} 606 \mathrm{~W}}$ & $q_{*, \mathrm{~F} 814 \mathrm{~W}}$ & $\begin{array}{c}\theta_{\mathrm{F} 606 \mathrm{~W}}^{\mathrm{a}} \\
(\mathrm{deg})\end{array}$ & $\begin{array}{c}\theta_{\mathrm{F} 814 \mathrm{~W}}{ }^{\mathrm{a}} \\
(\mathrm{deg})\end{array}$ \\
\hline Fred ….................. & $0.62 \pm 0.06$ & $0.69 \pm 0.07$ & $0.95 \pm 0.01$ & $0.94 \pm 0.01$ & $39 \pm 3$ & $26 \pm 2$ \\
\hline Ginger................. & $0.64 \pm 0.06$ & $0.66 \pm 0.07$ & $0.76 \pm 0.01$ & $0.79 \pm 0.01$ & $34 \pm 1$ & $33 \pm 1$ \\
\hline
\end{tabular}

${ }^{\text {a }}$ Position angles are measured north through east.

TABLE 5

SPeCtroscopic OBSERVATIONS

\begin{tabular}{|c|c|c|c|c|c|c|c|c|c|}
\hline Date & Target & Telescope & Instrument & Grating & $\begin{array}{c}\text { Wavelength Coverage } \\
\AA\end{array}$ & $\begin{array}{l}\text { Slit Width } \\
(\operatorname{arcsec})\end{array}$ & $\begin{array}{l}\text { Slit P.A. } \\
\text { (deg) }\end{array}$ & $\begin{array}{l}t_{\exp } \\
(\mathrm{s})\end{array}$ & $\mathrm{S} / \mathrm{N}^{\mathrm{a}}$ \\
\hline 2001 Jul 23 ............. & Fred & Keck II & ESI & $175^{\mathrm{b}}$ & $3887-10741$ & 1.0 & +11.8 & 3600 & 2.5 \\
\hline 2005 Apr $12 \ldots \ldots \ldots \ldots$ & Ginger & Keck I & LRIS/R & $600 / 5000$ & $4899-7488$ & 1.0 & +48.2 & 1800 & 8 \\
\hline 2005 Apr $13 \ldots \ldots \ldots \ldots$ & Fred & Keck I & LRIS/R & $600 / 5000$ & $4908-7452$ & 1.0 & +8.2 & 1800 & 8 \\
\hline 2005 May $13 . . . \ldots \ldots . . .$. & Fred & Gemini-N & GMOS & B 600 & $4587-7478$ & 1.0 & +8.2 & 4500 & 12 \\
\hline
\end{tabular}

${ }^{\text {a }}$ Value given is the average $\mathrm{S} / \mathrm{N}$ pixel ${ }^{-1}$ in the region just redward of the Ca II absorption lines.

b Cross-dispersed. 


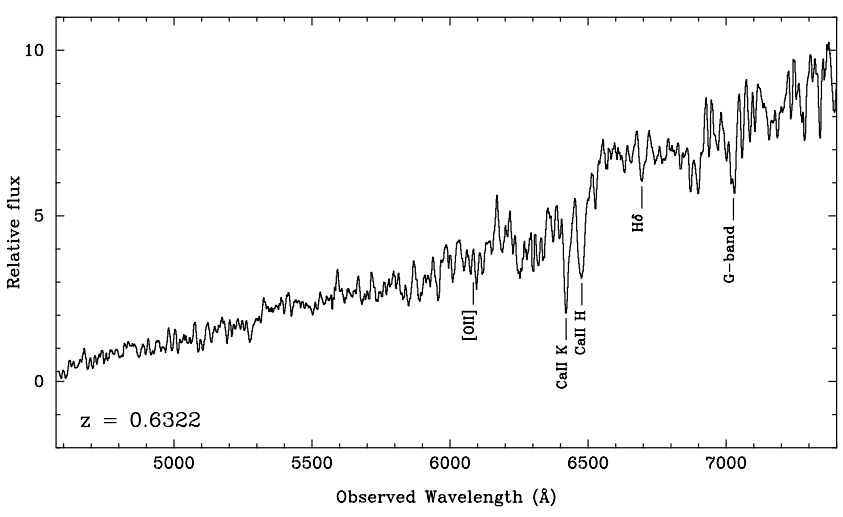

FIG. 3.-Optical spectrum of the Fred lens candidate, obtained with GMOS. The GMOS spectrum has been smoothed with a boxcar of width $9.2 \AA$ ( 5 pixels). The observed absorption features, as well as the expected location of the [O II] emission, have been marked.

excellent conditions, with a dark sky and seeing of $\sim 00^{\prime \prime} 6$. The three GMOS exposures of the system were dithered in the spectral direction in order to fill in the gaps between the chips.

The LRIS and ESI data were reduced using scripts that provided interfaces to the standard IRAF tasks. In addition, a portion of the ESI data reduction was performed using custom IDL scripts. The GMOS data were reduced using the gmos package in IRAF. All of the instrument-specific scripts performed the same procedures on the data. First, the bias level was subtracted using the overscan region of the chip. Next, pixel-to-pixel variations were corrected through division by a normalized flat-field frame that had been constructed by co-adding internal flat exposures taken immediately after the science exposures. The wavelength calibration for all of the exposures was based on arc lamp spectra obtained adjacent in time to the science observations. The wavelength solutions were used to straighten the sky lines and then sky subtraction was performed on the individual science spectra. The resulting spectra were co-added, after being weighted by the squares of their signal-to-noise ratios $(\mathrm{S} / \mathrm{Ns})$, to produce a final two-dimensional spectrum. The one-dimensional spectra shown in Figures 3-5 were each extracted from the appropriate final two-dimensional spectrum. Because the goal of the spectroscopy was simply to obtain redshifts, no correction for the response of the chip was performed.

The first lens candidate, Fred, has a redshift of $z_{\text {lens }}=0.6321$, based on multiple absorption features ( $\mathrm{Ca}$ II, $\mathrm{H} \delta$, and the $\mathrm{G}$ band). The GMOS spectrum is shown in Figure 3. The lower S/N ESI

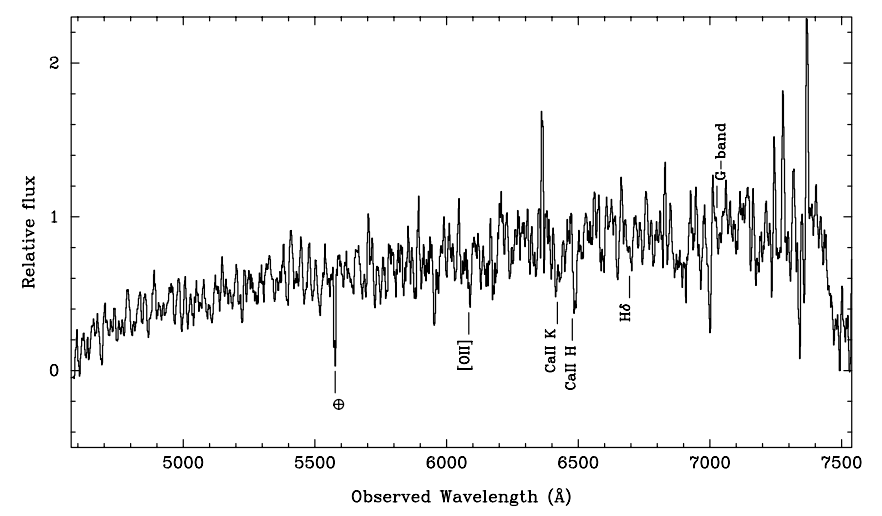

FIG. 4.-Optical spectrum of the Fred background object, obtained with GMOS. All of the marked features are associated with the lensing galaxy. The emission lines correspond to regions in which bright night-sky lines have been imperfectly subtracted. No clear emission lines from the background source are seen.

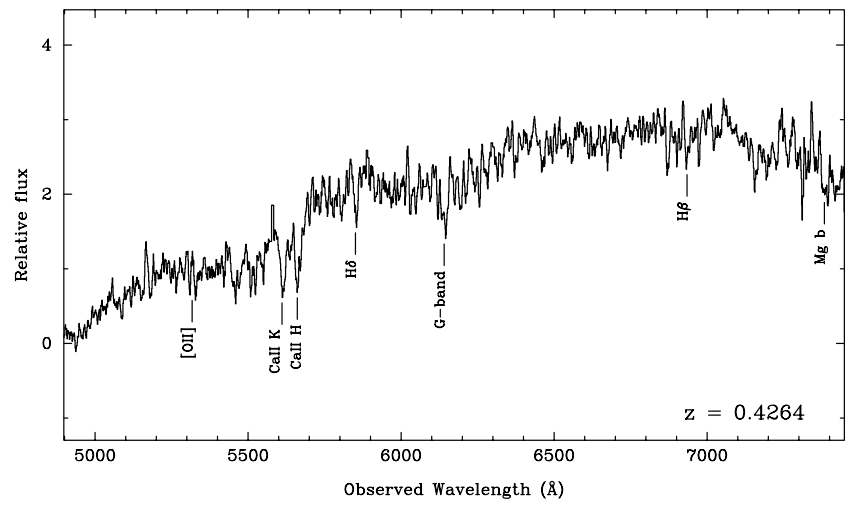

FIG. 5.-Optical spectrum of the Ginger lens candidate taken with LRIS on the Keck Telescope. The data have been smoothed with a boxcar of width 5 pixels, corresponding to $6.25 \AA$. The spectrum is dominated by the emission from the lens, whose spectral shape is consistent with an early-type galaxy. The redshift of the lens is $z=0.4264$. There is no evidence of ongoing star formation (the expected position of the $[\mathrm{O} \mathrm{II}]$ emission line is marked).

and LRIS spectra show the same features. The Fred redshift places it in a small group that also includes the lensing galaxy in the B1608+656 system (Fassnacht et al. 2006). The ESI observations were obtained before the system was identified as a lens candidate. Although the position angle (P.A.) of the slit was approximately correct for the system morphology, no trace of the lensed source is seen in the spectrum. On the other hand, the GMOS spectrum of this object was obtained with a slit P.A. chosen specifically to cover both the lensing galaxy and the lensed source. The brighter of the two blue blobs is clearly seen in the two-dimensional spectrum and is spatially separated from the lens galaxy spectrum, allowing a separate spectrum to be extracted for the lensed image (Fig. 4). However, no clear emission or absorption features, beyond those due to contamination from the lens galaxy light and imperfect subtraction of the night-sky lines, are seen in the spectrum of the source. Given the blue color of the lensed source, we expect to see emission lines in the spectrum; the lack of emission features allows us to place tentative limits on the redshift of the background source. Emission from [O II] $\lambda 3727$ shifts out of the range covered by the GMOS spectrum at a redshift of $z_{\text {src }}=1.0$, while Ly $\alpha$ emission enters the spectral range at $z_{\mathrm{src}}=2.8$. We therefore assume that the background object falls within this redshift range. For completeness we have to consider the possibility that the blue knots are star formation associated with the lensing galaxy. However, if this were the case, we would expect strong [O II] emission at a wavelength of $6083 \AA$. We do not see any evidence for [O II] emission at $6083 \AA$ in any of the Fred spectra and therefore conclude that the arc is not due to star formation in the lensing galaxy.

The other lens candidate, Ginger, has a spectrum typical of an early-type galaxy (Fig. 5). A number of absorption features, including lines due to $\mathrm{Ca}$ II $\mathrm{H}$ and $\mathrm{K}, \mathrm{H} \delta$, and the $\mathrm{G}$ band, give a redshift of $z_{\text {lens }}=0.4264$. Although the slit P.A. was chosen to cover both the galaxy and the brightest portion of the arc, no redshift for the background source was obtained. This may just be due to the short integration time and bright night sky. The redshift of the lens galaxy places it in another group detected along the line of sight to B1608+656. This group has a mean redshift of $z=0.426$ (Fassnacht et al. 2006).

\section{GRAVITATIONAL LENS MODELS}

We reconstruct the lensed images and source of both systems to test the lens hypothesis and assess whether a relatively simple 

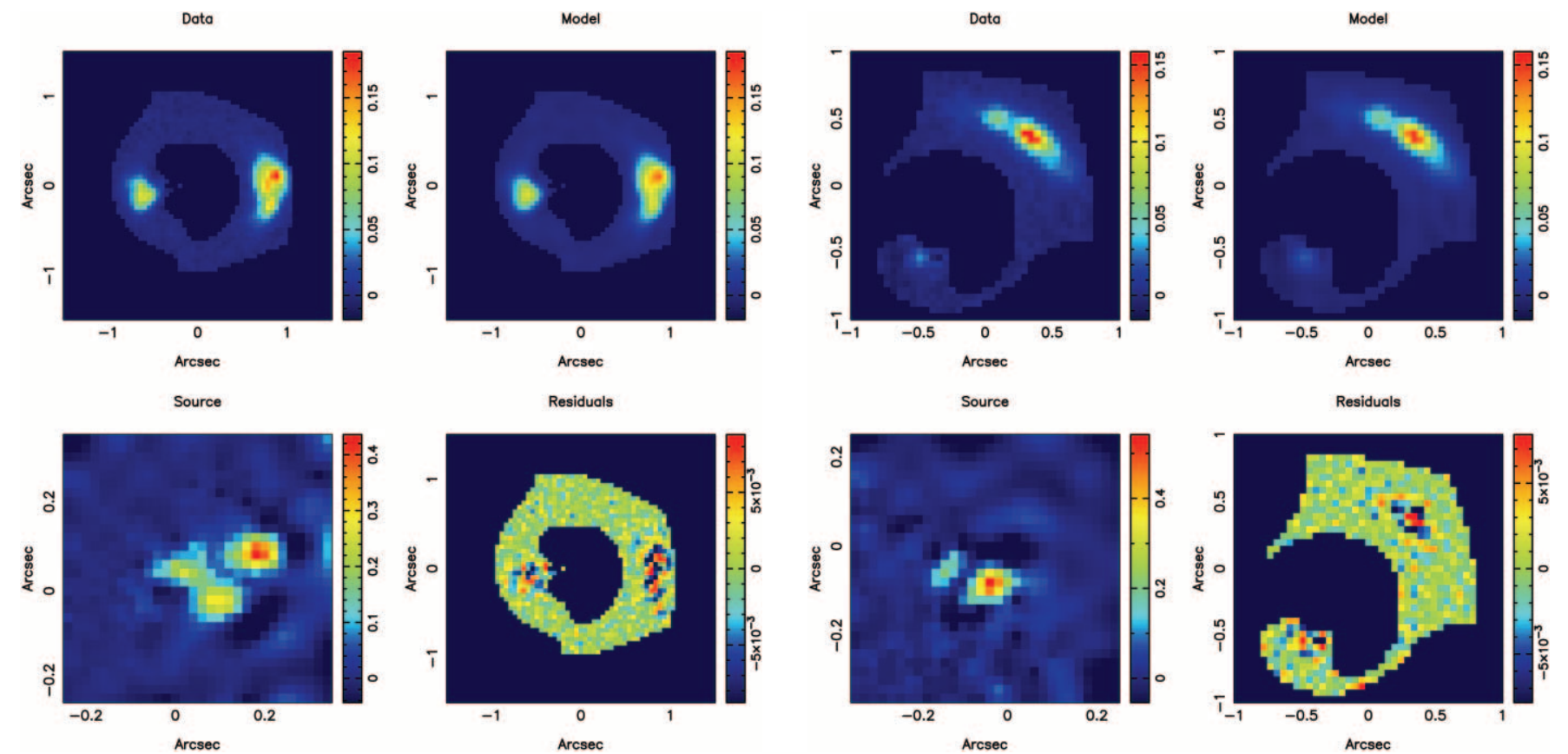

FIG. 6.-Left: Model results for the Fred lens candidate. Panels show observed data with lensing galaxy subtracted (top left), the reconstructed lensed image (top right), the

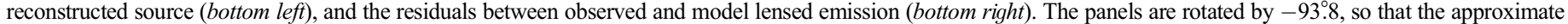

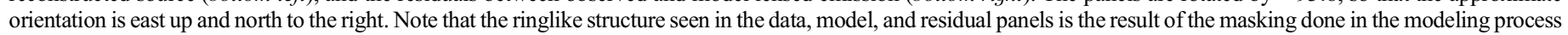
and does not represent an Einstein ring. Right: Idem, but for the Ginger lens candidate.

mass model can explain the observed lenslike features in Figure 1. We adopt a singular isothermal ellipsoid (SIE) lens mass model (Kormann et al. 1994), which describes the mass distribution (stellar plus dark matter) in the inner regions of massive lens galaxies extremely well (e.g., Treu \& Koopmans 2004; Koopmans et al. 2006). In addition, we add where necessary an external shear to account for the environment of the lenses (see $\S \S 5.1$ and 5.2), motivated by the discovery of several galaxy groups along this line of sight (Fassnacht et al. 2006). The modeling is done via a nonparametric reconstruction method, which is described in more detail in Treu \& Koopmans (2004) and Koopmans (2005). We regularize the solutions somewhat to attain a smoother solution; see Koopmans (2005) for a proper discussion. The modeling is conducted on the F606W ACS images, after subtracting off the emission from the lensing galaxy. The galaxy-subtracted images are shown in the top left panels of Figure 6. The Fred image shows clearly the two bright lensed images. In the Ginger image, the arc is seen in the top right corner of the galaxy-subtracted image, while there is a hint of a counterimage in the lower left corner.

The resulting nonparametric source and lens models are shown in Figure 6 for both systems, where the mass models in each case are centered on the brightness peaks of the observed galaxies. The resulting SIE and external shear parameters, defined as in Kormann et al. (1994), are given in Table 6. The "lens

TABLE 6

Lens Model Parameters

\begin{tabular}{|c|c|c|}
\hline Parameter & Fred & Ginger \\
\hline$b_{\text {SIE }}(\operatorname{arcsec}) .$. & 0.73 & 0.61 \\
\hline$\theta_{\mathrm{SIE}}(\mathrm{deg}) \ldots \ldots$ & $\cdots$ & {$[+34]^{\mathrm{a}}$} \\
\hline 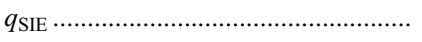 & {$[1.0]^{\mathrm{a}}$} & 0.84 \\
\hline$\gamma_{\text {ext }} \ldots \ldots \ldots \ldots \ldots \ldots$ & 0.056 & $\cdots$ \\
\hline$\theta_{\text {ext }}(\operatorname{deg}) \ldots \ldots$ & +225 & $\ldots$ \\
\hline
\end{tabular}

${ }^{\text {a }}$ Held fixed at observed value. strength," $b_{\text {SIE }}$, is defined such that the mass enclosed by the critical curve is independent of the ellipticity. For a singular isothermal sphere, $b_{\text {SIE }}$ corresponds to the Einstein ring angular radius. The external shear P.A., $\theta_{\text {ext }}$, points in the direction of the mass producing the shear. Both $\theta_{\mathrm{SIE}}$ and $\theta_{\mathrm{ext}}$ are defined in the standard astronomical convention, measured counterclockwise from the north axis. Note, however, that image frames in Figure 6 are rotated by $-93^{\circ} .8$ with respect to the sky and therefore the position angles in the table do not correspond to the orientations in the figure. The bracketed quantities in the table are fixed at the observed values.

\section{DISCUSSION}

The first question to address is whether or not the candidates presented in this paper are actually gravitational lenses. Of course, a measurement of the redshifts of the background objects would make the lens hypothesis more secure. The current set of spectra were obtained with limited observing time or wavelength coverage or were observed with the slit at a nonoptimal P.A. Therefore, a more dedicated observing campaign may yet yield the redshifts, especially at shorter wavelengths where the contrast between the lens and the source is improved (as for HST 1543; see Treu \& Koopmans 2004). The blue colors of the background objects suggest that their spectra may contain emission lines and thus that it may be possible to obtain redshifts in spite of the faintness of the objects. Even without redshifts, however, the system morphologies and the surface brightnesses of the background objects are consistent with gravitational lensing, as can be seen from the lens modeling.

\subsection{The Lens Model for Fred}

Based on several arguments, besides the similar colors and typical lens geometry of the two galaxy-subtracted residual images, we strongly believe that Fred is a second strong-lens system in the field of the B1608+656 lens system: (1) A simple smooth 
lens mass model, with only small perturbations from the nonparametric modeling, is able to reproduce the observed image brightness distributions extremely well, with small residuals between the observed images and the lens model. The reconstructed source correctly maps onto the triangular structure seen in both lensed images. The source has an unusual morphology, but, as we argue below, this is not unexpected. The fact that both lensed images show the same unusual morphology, with the proper parity flip, only strengthens the case that this system is a lens. (2) The lens strength of $b_{\mathrm{SIE}}=0.73$, determined from the bestfit model, implies an approximate stellar velocity dispersion $\sigma_{\text {SIE }}$ between 290 and $210 \mathrm{~km} \mathrm{~s}^{-1}$ for source redshifts between $z_{\text {src }}=1.0$ and 2.0, respectively. These values are typical for most lens galaxies (i.e., around $L_{*}$ ) and also agree well with the brightness of the galaxy and absence of emission lines in the spectra. A direct measurement of its velocity dispersion and source redshift, however, can secure this agreement more accurately. (3) The SIE model for Fred requires an external shear of $\gamma_{\mathrm{ext}} \approx 0.06$ with a P.A. of $225^{\circ}$. This is the direction of the previously known lens system, B1608+656 (see Fig. 2), and could be caused by its group environment (Fassnacht et al. 2006), although the group parameters are not well constrained by the current data. (4) The last piece of evidence (circumstantial in this case) is the good agreement between the ellipticity of the stellar light and that of the SIE mass model. This correlation is typically very strong (rms of $\sim 0.1$ in $q_{*} / q_{\text {SIE }}$; see Koopmans et al. 2006) for elliptical galaxy systems with a significant stellar mass component inside the Einstein radius.

The morphology of the lensed source is unusual. However, given its blue color and presumed (i.e., relatively high) redshift, one might expect the emission from such a galaxy to be dominated by knots of star formation. In fact, the source shape is similar to several of the reconstructed lensed sources in the Sloan Lens ACS Survey (SLACS) sample of Bolton et al. (2006). Generally speaking, the increased abundance of peculiar and irregular galaxies at high redshifts and faint magnitudes is well established from deep surveys (e.g., Abraham et al. 1996). Once again, the facts that the unusual morphology is seen in both components and that the lens model so clearly maps the brighter image into the fainter one are strong arguments in favor of the lensing hypothesis.

The projected mass of the lensing galaxy, within the critical curve, is given by

$$
M_{\mathrm{E}}=\frac{c^{2}}{4 G} \frac{D_{l} D_{s}}{D_{l s}} b_{\mathrm{SIE}}^{2},
$$

where $D_{l}, D_{s}$, and $D_{l s}$ are the angular diameter distances to the lens, to the background source, and between the lens and the background source, respectively. The definition of $b_{\text {SIE }}$ is such that the above equation is correct, independent of the axis ratio (e.g., Treu \& Koopmans 2004). The derived mass of the lensing galaxy is $6.9 \times 10^{10}\left(D_{s} / D_{l s}\right) h^{-1} M_{\odot}$. For source redshifts between 1.0 and 2.0, this corresponds to masses between $2.2 \times 10^{11}$ and $1.1 \times 10^{11} h^{-1} M_{\odot}$. Adopting the surface photometry derived in $\S 2.1$, this corresponds to a $B$-band mass-to-light ratio of $13.6 h(6.8 h)$ in solar units inside the cylinder of radius equal to the Einstein ring radius, for $z_{\mathrm{src}}=1.0(2.0)$. These values are consistent with those found for other lens galaxies (e.g., Treu \& Koopmans 2004), lending further support to the lensing hypothesis for Fred.

The velocity dispersion estimated via the FP in $\S 2.1$ provides a further consistency check on the lensing interpretation, or an estimate of the redshift of the background source if the lensing hypothesis is accepted (e.g., Kochanek et al. 2000). In fact, empirical evidence suggests that at scales comparable to the effective radius, the ratio of stellar to SIE velocity dispersion, $f_{\mathrm{SIE}}=\sigma_{*} / \sigma_{\mathrm{SIE}}$, is close to unity (e.g., Kochanek et al. 2000; van de Ven et al. 2003; Treu \& Koopmans 2004; Treu et al. 2006; Koopmans et al. 2006). The estimated velocity dispersion from the $\mathrm{FP}\left(\sigma_{\mathrm{FP}}=\right.$ $180 \pm 50 \mathrm{~km} \mathrm{~s}^{-1}$ ) is consistent with that of the best-fitting SIE for plausible $z_{\text {src }}$, consistent with the lensing hypothesis. Conversely, adopting the lens hypothesis, $\sigma_{\mathrm{FP}}$ would imply $z_{\mathrm{src}}>1.52$ for $f_{\mathrm{SIE}}=1.0 \pm 0.1$.

\subsection{The Lens Model for Ginger}

This system is significantly less constrained because the presumed counterimage is very faint and the brightness distribution of the images lacks very distinct structure. Due to the lack of constraints, only the lens strength $\left(b_{\mathrm{SIE}}\right)$ and ellipticity $\left(q_{\mathrm{SIE}}\right)$ are varied in the modeling, and no external shear is used. The resulting reconstructed source consists of two features that correspond to those seen in the brighter of the two (lensed) images. Even though both the geometry and the relative brightnesses of the images can be fitted by a model with several free parameters, the models are very tentative. The lens strength of $b_{\mathrm{SIE}}=0.61$, determined from the best-fit model, implies an approximate stellar velocity dispersion between 205 and $180 \mathrm{~km} \mathrm{~s}^{-1}$ for source redshifts between $z_{\text {src }}=1.0$ and 2.0, respectively. Following the same arguments as for Fred, we can obtain consistency checks or an estimate for $z_{\text {src }}$, by comparing the results of the lens model with the surface photometry. The lens strength implies a mass within the cylinder of $7.3 \times 10^{10} h^{-1} M_{\odot}\left(5.4 \times 10^{10} h^{-1} M_{\odot}\right)$ for $z_{\text {src }}=1.0(2.0)$. This corresponds to a $B$-band mass-to-light ratio of $11.4 h(8.4 h)$ in solar units for $z_{\text {src }}=1.0(2.0)$, which is consistent with typical values found for other lens galaxies at similar redshift (Koopmans et al. 2006). The velocity dispersion implied by the FP, $\sigma_{\mathrm{FP}}=140 \pm 30 \mathrm{~km} \mathrm{~s}^{-1}$, is somewhat (but not significantly for the higher source redshift range) smaller than that obtained from the lens model for $z_{\text {src }}$ in the range $1-2$. Thus, under the lens hypothesis either $z_{\text {src }}>2.2$ is required $\left(z_{\mathrm{Src}}>3\right.$ for $\left.f_{\mathrm{SIE}}=1.0 \pm 0.1\right)$ or $f_{\mathrm{SIE}}<1$, possibly indicating extra convergence from the environment of Ginger. None of these arguments appear conclusive as to the lensing nature of Ginger. Whether Ginger is a third strong lens in the field of B1608+656 will probably require a direct measurement of the source redshift and a more definitive detection of the counterimage.

\section{SUMMARY AND CONCLUSIONS}

Our investigations have shown that the single ACS pointing centered on B1608+656 contains two additional strong lens candidates. This result implies that there are one to two additional lenses in a $\sim 10 \operatorname{arcmin}^{2}$ area of the sky, giving a posteriori lensing rates of $0.07-0.46 \mathrm{arcmin}^{-2}$ if only Fred is a real lens, and $0.14-0.59 \mathrm{arcmin}^{-2}$ if both of the candidates are real lenses (68\% limits assuming Poisson statistics; Gehrels 1986). These rates should be contrasted with the results of other HST lens search campaigns, which have found lower lensing rates. For example, 10 lens candidates, two of which have been confirmed, were found in the $\sim 600 \mathrm{arcmin}^{2}$ of the Medium Deep Survey (Ratnatunga et al. 1999), for a lensing rate of $\leq 0.02 \mathrm{arcmin}^{-2}$. Also, the search in the Great Observatories Origins Deep Survey (GOODS) ACS data by Fassnacht et al. (2004) resulted in six lens candidates in $\sim 300 \mathrm{arcmin}^{2}$, once again giving a lensing rate of at most $\sim 0.02 \mathrm{arcmin}^{-2}$.

In hindsight, it might not be surprising that the lensing rate in the B1608+656 field is an order of magnitude higher than those 
in the larger surveys, although the effect of small number statistics should not yet be discounted. Qualitatively this is easy to understand because the field that is being imaged in these observations is not a random line of sight. First, the targeted field is already known to contain a massive early-type lens galaxy. These galaxies often are found in dense environments such as groups and clusters (e.g., Dressler 1980). Spectroscopic investigations of this particular field have revealed the presence of at least three galaxy groups along the line of sight to the B1608+656 lens system, including a group that is physically associated with the lensing galaxy (Fassnacht et al. 2006). Second, the lensed source in the B1608+656 system is itself a massive early-type galaxy exhibiting AGN activity in the radio domain (Myers et al. 1995; Fassnacht et al. 1996). Both as a massive galaxy and as a radio source (e.g., Allington-Smith et al. 1993), the background source can be expected to reside in an overdense environment. The lensed object in the B1608+656 system is at a redshift of $z_{\text {src }}=1.394$ (Fassnacht et al. 1996). If the background sources in Fred and Ginger were at the same redshift, their angular separations from the B1608+656 system would correspond to $\sim 210 \mathrm{~h}^{-1} \mathrm{kpc}$, not unusual for a group of galaxies. Third, by obtaining much deeper than normal space-based imaging of this field, we have started to pick up the ubiquitous population of faint blue galaxies. Depending on the steepness of the luminosity function of this population of galaxies, magnification bias can lead to a higher lensing rate than expected based on the density of galaxies at the limiting magnitude of the imaging (Bolton et al. 2006).

Being quantitative about the expected lensing rate is rather more difficult because it depends on many unknown factors such as the redshift and mass distributions of the potential lensing galaxies and the redshift distribution of the sources, and thus it is beyond the scope of this paper. However, to test the plausibility of the qualitative argument, we take a simple path to estimate the expected lensing rate in a deep ACS image such as this one. Our assumptions are that (1) only early-type galaxies will act as lensing galaxies and (2) all of the lenses will have lensing cross sections of $\sim 1 \operatorname{arcsec}^{2}$. The lensing cross section is estimated by taking a circular area with a diameter equal to the typical image separation for the lenses discovered by the Cosmic Lens AllSky Survey (Browne et al. 2003). We define the early-type galaxies as luminous $(\mathrm{F} 814 \mathrm{~W}<21.5)$ red $(\mathrm{F} 606 \mathrm{~W}-\mathrm{F} 814 \mathrm{~W}>$ 1.0) galaxies that also have morphologies typical of early types (i.e., excluding galaxies with clear disklike structure). As a sanity check, all three of the lensing galaxies in this field satisfy the above criteria. The magnitude, color, and morphology cuts yield a conservative estimate of 14 potential lenses. To test the validity of our cuts, we extended them to 1 mag fainter and 1 mag bluer. Of the 30 additional galaxies that were thus included, only two satisfied the morphology criterion. Therefore, we assume that our cuts have located the vast majority of the luminous elliptical galaxies in the field. The resulting total cross section for lensing in this field is $\sim 16 \mathrm{arcsec}^{2}$. This cross section must be compared to the density of faint blue galaxies. The completeness limit for the F606W imaging that is presented in this paper is $\mathrm{F} 606 \mathrm{~W} \sim 26.5 .{ }^{9}$ Assuming a typical magnification factor from strong lensing of a few, which is what we find from the lens models, we thus require a knowledge of the integrated number density of galaxies with F606W $<28$. We use the corrected number counts of Benitez

\footnotetext{
9 Here the magnitudes are in the $\mathrm{AB}$ system, in order to compare to the results of Benitez et al. (2004) .
}

et al. (2004) to obtain an integrated number density of $\sim 2 \times$ $10^{6}$ galaxies $\mathrm{deg}^{-2}$ or $\sim 0.2$ galaxies $\operatorname{arcsec}^{-2}$. The combination of this surface density with the approximate lensing cross section in the field yields an a posteriori expectation value of $3 \pm 2$ lenses, given our assumptions. Therefore, it appears quite likely that two additional lenses would be found in these images.

We conclude that an efficient method to search for new gravitational lenses is to obtain deep images of the fields surrounding known strong lenses. This method takes advantage of the enhancement of the line-of-sight number densities of both the potential lenses and potential sources. We note that this is more an effect of biased galaxy formation than a bias due to the enhancement of the lensing cross section by the group environment, which is a secondary effect to lensing statistics (e.g., Keeton \& Zabludoff 2004; Fassnacht et al. 2006). We also note that the results presented here suggest that clustering of sources and lenses should be taken into account when comparing observed and predicted lensing statistics in order, e.g., to place limits on cosmological parameters.

These observations would not have been possible without the expertise and dedication of the staffs of the Palomar and Keck observatories. We especially thank Karl Dunscombe, Grant Hill, Jean Mueller, Gary Puniwai, Kevin Rykoski, Gabrelle Saurage, and Skip Staples. We thank the anonymous referee for timely comments that led to improvements to the paper. C. D. F. and J. P. M. acknowledge support under HST program GO-10158. Support for program GO-10158 was provided by NASA through a grant from the Space Telescope Science Institute, which is operated by the Association of Universities for Research in Astronomy, Inc., under NASA contract NAS 5-26555. This work is supported in part by the European Community's Sixth Framework Marie Curie Research Training Network Programme, contract MRTN-CT-2004-505183 "ANGLES." Based in part on observations made with the NASA/ESA Hubble Space Telescope, obtained at the Space Telescope Science Institute, which is operated by the Association of Universities for Research in Astronomy, Inc., under NASA contract NAS 5-26555. These observations are associated with program GO-10158. Some of the data presented herein were obtained at the W. M. Keck Observatory, which is operated as a scientific partnership among the California Institute of Technology, the University of California, and the National Aeronautics and Space Administration. The Observatory was made possible by the generous financial support of the W. M. Keck Foundation. The authors wish to recognize and acknowledge the very significant cultural role and reverence that the summit of Mauna Kea has always had within the indigenous Hawaiian community. We are most fortunate to have the opportunity to conduct observations from this mountain. Based on observations obtained at the Gemini Observatory, which is operated by the Association of Universities for Research in Astronomy, Inc., under a cooperative agreement with the NSF on behalf of the Gemini partnership: the National Science Foundation (United States), the Particle Physics and Astronomy Research Council (United Kingdom), the National Research Council (Canada), CONICYT (Chile), the Australian Research Council (Australia), CNPq (Brazil), and CONICET (Argentina).

Facilities: HST (ACS), Keck:I (LRIS), Keck:II (ESI), Gemini: Gillett (GMOS-North), PO:1.5m (CCD13) 
REFERENCES

Abraham, R. G., Tanvir, N. R., Santiago, B. X., Ellis, R. S., Glazebrook, K., \& van den Bergh, S. 1996, MNRAS, 279, L47

Allington-Smith, J. R., Ellis, R., Zirbel, E. L., \& Oemler, A. J. 1993, ApJ, 404, 521

Bahcall, J. N., Maoz, D., Doxsey, R., Schneider, D. P., Bahcall, N. A., Lahav, O., \& Yanny, B. 1992, ApJ, 387, 56

Benitez, N., et al. 2004, ApJS, 150, 1

Bertin, E., \& Arnouts, S. 1996, A\&AS, 117, 393

Blakeslee, J. P., et al. 2004, ApJ, 602, L9

Bolton, A. S., Burles, S., Koopmans, L. V. E., Treu, T., \& Moustakas, L. A. 2005, ApJ, 624, L21 2006, ApJ, 638, 703

Bolton, A. S., Burles, S., Schlegel, D. J., Eisenstein, D. J., \& Brinkmann, J. 2004, AJ, 127, 1860

Browne, I. W. A., et al. 2003, MNRAS, 341, 13

Bruzual, A., G., \& Charlot, S. 1993, ApJ, 405, 538

Djorgovski, S. G., \& Davis, M. 1987, ApJ, 313, 59

Dressler, A. 1980, ApJ, 236, 351

Dressler, A., Lynden-Bell, D., Burstein, D., Davies, R. L., Faber, S. M., Terlevich, R., \& Wegner, G. 1987, ApJ, 313, 42

Fassnacht, C. D., Gal, R. R., Lubin, L. M., McKean, J. P., Squires, G. K., \& Readhead, A. C. S. 2006, ApJ, 642, 30

Fassnacht, C. D., Moustakas, L. A., Casertano, S., Ferguson, H. C., Lucas, R. A., \& Park, Y. 2004, ApJ, 600, L155

Fassnacht, C. D., Womble, D. S., Neugebauer, G., Browne, I. W. A., Readhead, A. C. S., Matthews, K., \& Pearson, T. J. 1996, ApJ, 460, L103

Ford, H., et al. 1998, Proc. SPIE, 3356, 234 2003, Proc. SPIE, 4854, 81

Gehrels, N. 1986, ApJ, 303, 336

Hook, I. M., Jørgensen, I., Allington-Smith, J. R., Davies, R. L., Metcalfe, N., Murowinski, R. G., \& Crampton, D. 2004, PASP, 116, 425

Keeton, C. R., \& Zabludoff, A. I. 2004, ApJ, 612, 660

Kochanek, C. S. 2006, in Gravitational Lensing: Strong, Weak and Micro, ed. G. Meylan, P. Jetzer, \& P. North (Berlin: Springer), 91

Kochanek, C. S., Keeton, C. R., \& McLeod, B. A. 2001, ApJ, 547, 50
Kochanek, C. S., et al. 2000, ApJ, 543, 131

Koekemoer, A. M., Fruchter, A. S., Hook, R. N., \& Hack, W. 2002, in The 2002 HST Calibration Workshop: Hubble after the Installation of the ACS and the NICMOS Cooling System, ed. S. Arribas, A. Koekemoer, \& B. Whitmore (Baltimore: STScI), 337

Koopmans, L. 2005, in Baryons in Dark Matter Halos, ed. R. Dettmar, U. Klein, \& P. Salucci (SISSA Proceedings of Science), 66

Koopmans, L. V. E., Treu, T., Bolton, A. S., Burles, S., \& Moustakas, L. A. 2006, ApJ, 649, 599

Koopmans, L. V. E., Treu, T., Fassnacht, C. D., Blandford, R. D., \& Surpi, G. 2003, ApJ, 599, 70

Kormann, R., Schneider, P., \& Bartelmann, M. 1994, A\&A, 284, 285

Monet, D., et al. 1998, USNO-A2.0 Catalogue (Washington DC: US Naval Obs.)

Myers, S. T., et al. 1995, ApJ, 447, L5 2003, MNRAS, 341, 1

Oke, J. B., et al. 1995, PASP, 107, 375

Peng, C. Y., Ho, L. C., Impey, C. D., \& Rix, H.-W. 2002, AJ, 124, 266

Pindor, B., et al. 2004, AJ, 127, 1318

Ratnatunga, K. U., Griffiths, R. E., \& Ostrander, E. J. 1999, AJ, 117, 2010

Sheinis, A. I., Bolte, M., Epps, H. W., Kibrick, R. I., Miller, J. S., Radovan, M. V., Bigelow, B. C., \& Sutin, B. M. 2002, PASP, 114, 851

Thuan, T. X., \& Gunn, J. E. 1976, PASP, 88, 543

Treu, T., \& Koopmans, L. V. E. 2004, ApJ, 611, 739

Treu, T., Koopmans, L. V. E., Bolton, A. S., Burles, S. C., \& Moustakas, L. A. 2006, ApJ, 640, 662

Treu, T., Stiavelli, M., Møller, P., Casertano, C., \& Bertin, G. 2001, MNRAS, 326,221

Treu, T., et al. 2005, ApJ, 633, 174

van de Ven, G., van Dokkum, P. G., \& Franx, M. 2003, MNRAS, 344, 924

Willis, J. P., Hewett, P. C., Warren, S. J., Dye, S., \& Maddox, N. 2006, MNRAS, 369, 1521

Winn, J. N., et al. 2000, AJ, 120, 2868

Wisotzki, L., Schechter, P. L., Bradt, H. V., Heinmüller, J., \& Reimers, D. 2002 , A\&A, 395, 17 